\title{
COOPERATIVE DEFENSE FIREWALL PROTOCOL
}

\author{
Magdi M. S. El-Soudani " and Mohamed A. Eissa ${ }^{b}$ \\ ${ }^{a}$ National Telecommunications Institute (NTI), MCIT, Egypt, melsoudani@menanet.net \\ ${ }^{\mathrm{b}}$ Intel Corp., Canada, meissa3560@ rogers.com
}

Abstract: Most of today's firewalls are standalone systems. This fact leads to some limitations in defending organized multi-point attacks. Therefore, communicating firewalls were introduced. This opens the door for a cooperative defending technique, capable of pushing and isolating attacks far from the targets. In this work, we propose a new firewall communication protocol that fulfills the cooperative defense model. In this protocol, a firewall node broadcasts its security policy to the surrounding firewall nodes. The security policy travels in the path towards the point of attack, so the firewall nodes enforce the security policy and cooperate in defending the targets and isolating the attack to the closest possible point to the source of attack. The security of the communication protocol is studied to ensure its strength against some network threats and to protect the network from using the protocol itself in denial of service attacks. The behavior of the protocol is studied using a protocol simulator. Using this simulator, we execute different experiments to observe the protocol behavior.

Keywords: Communication Firewall, Denial of Service, Border Gateway protocol.

\section{INTRODUCTION}

Smith and Bhattacharya in their 1999 paper [1] have proposed a Communicating Gateway Firewall Protocol (CGFW) as a tool for a distributed defending model. The main objective of that model is to stop the Denial of Service (DoS) attack, which is one of the major attacks on the Internet [2],[3]. The idea was to distribute firewalls across the Internet and to develop the firewall to be active in case of attack. Usually the firewall is placed on the perimeter of the network. The CGFW was built on top of one of routing protocol, the Border Gateway Protocol 4 (BGP-4).The CGFW uses BGP-4 as a transporting protocol and saves the cost of implementation

The original version of this chapter was revised: The copyright line was incorrect. This has been corrected. The Erratum to this chapter is available at DOI: 10.1007/978-0-387-35691-4_52 
from scratch [4]. Its main inefficiency is the fact that BGP-4 was designed to be a routing protocol and not a firewall communication protocol. In the following section, we briefly discuss their approach and its limitations. Afterwards, we present the proposed new cooperative defense firewall protocol (CDFP). The design objectives and assumptions are also considered in section three. The security of the protocol itself is discussed as well as the protection of the network from the denial of service attack using CDFP itself. An example is given in section four to describe the behavior of the protocol during an attack. We conclude our work with a comparison between the CGFW and the proposed CDFP.

\section{DISTRIBUTED COMMUNICATING FIREWALLS PROTOCOL}

The CGFW communicating firewall protocol [1] is a distributed defending model that is used to stop the Denial of Service (DoS) attack. The idea was to distribute firewalls across the Internet and to develop the firewall to be active in case of attack. In CGFW protocol, the originator requester is typically a node that has been under attack and wants to block the attack in a point nearer to the source of the attack. However, any CGFW node can initiate the protocol with any other CGFW node on behalf of the originator. In other words, any node can invoke other nodes in the effort to push the attacker near to the source of the attack. Since the CGFW is based on BGP-4 routing protocol, its disadvantages are mainly due to the limitations of BGP4 protocol, which does contain security measures. The protocol also needs to continuously maintain a table containing full details of the network topology. This table consumes huge storage space and long search time to retrieve the required information while calculating the optimum nodes to be contacted in the defending process. It does not utilize the capabilities of application level proxy firewall for example. There is no conflict resolution mechanism to resolve the problem of receiving contradicting security rules at a given firewall node which tries to cooperate in the defending process.

\section{COOPERATIVE DEFENSE FIREWALL PROTOCOL}

The main objective of the proposed protocol is to enable a group of firewall systems to communicate and to work together to stop the traffic generated by an attacker at a earlier point as possible. This is achieved by broadcast security policies between firewalls to isolate the attacks. The protocol to follows the changes in network topology and security policy. It has also to resist IP spoofing. In the design of the protocol we assume that all nodes are under the same administration (autonomous system), firewall nodes will cooperate in the defending process, and all firewall nodes are 
aware of the proposed firewall communication protocol prior to defending the network. We also assume that there is a certificate authority server (CA) in the network that is accessible by all nodes.

\subsection{CDFP Architecture}

A firewall automatically decides its role according to either the information it receives from foreign networks, or through its detection of violation of its own network security policy. Firewalls within our proposed system can take two major roles; they can either act as a Defender Firewall (DFW), or as an Assistant Firewall (AFW). A defender firewall is physically connected to the target network. The DFW inspects each incoming packet against its security policy database. If a violation occurs, it will create a "deny" security rule, add it to its policy database and mark it as active. It will also log the incident's details into the database for historical reporting, and notification of the administration. The protocol also introduces a new process that is scheduled to run every interval $\mathrm{T}_{\text {broadcast }}$. When this broadcast service is due, it will scan the policy database to extract all the entries marked as active. When an assistant firewall receives a broadcast message, it will inspect the message to make sure it has not been tampered. Then, it will extract the rules included in the message and check their security. If the message and the rules have proved to be valid, it will add them into its own policy database. Mark them as inactive initially and set their type as dynamic rules (DR). We can summarize the protocol rules as follows:

For "deny" rules:

- If "deny" rule is used, mark the rule as active and broadcast it to the surrounding nodes. When the rule is broadcasted mark it as inactive.

- Discard "deny" rule if it is inactive for time $t>=t_{d}$.

For "permit" rules:

- A "permit" rule is always broadcasted (always active) till it is discarded after time $\mathrm{t}=\mathrm{t}_{\mathrm{p}}$ or if "deny" rule reaches a matching "permit", and replaces it.

- If "permit" rule reaches a matching "deny", it will replace it.

For all rules:

- Never discard static rules unless stated by the administrator

- Split horizon technique can be used also, which means not to broadcast the information came from a certain direction to the same direction again. 


\subsection{Security Policy Changes}

Policy changes can occur from two major sources, administrative decisions, or decisions initiated by an Intrusion Detection System (IDS). These changes either permit denied rules, or deny allowed ones. If a "deny" rule was broadcasted previously to other firewalls, the traffic gets filtered from earlier nodes on the network. To change this, a "permit" rule must be created to reflect the policy change, and rebroadcasted again to the network in order for the change to take place.

The DFW is the source of policy changes. To change a "deny" rule, the DFW has to initiate a rule exactly the same as the previously issued denied rule, but with a "permit" action instead. Then the DFW broadcasts this rule to its neighbors, and by turn, they will do the same until the rule is permitted network wide.

\subsection{CDFP Protocol Security}

The proposed cooperative defense firewall protocol, like any other communication protocol can introduce new risks to the network, due to its existence in public, insecure media. The protocol would provide an attacker with a perfect tool to mount a Denial of Service (DoS) attack if he could alter/simulate the messages exchanged between firewalls to impose false policy. By achieving this, malicious intruders can deny a trusted node/user an authorized access to certain network resources. As examples of possible threats to CDFP are masquerading, message altering (Man-In-the-Middle attack - MIM), and message replay. To overcome these threats access control mechanism, authentication, integrity check are used. Based on RSA algorithm, a public-key certificate authority CA is also assumed tob part of the network. A given firewall node $n$ should have the following components available prior to issuing a message; a public/private key pair $\left(\mathrm{K}_{\mathrm{npb}}, \mathrm{K}_{\mathrm{npr}}\right)$ and a digital certificate $\mathrm{C}_{\mathrm{n}}$ issued by $\mathrm{CA}$.

Every firewall node generates a digital certificate enrollment request to $\mathrm{CA}$. This enrollment request contains the public key of the firewall node, as well as its identity information, such as the nodes public name, its IP address, DNS name, etc. We also include the IP addresses and netmasks of the networks, which this firewall is held responsible for, within the request. After the verification process held by the $\mathrm{CA}$ concludes, the $\mathrm{CA}$ will approve and issue a digital certificate $C_{n}$ for that node, containing information that verifies the identity of this firewall, and describes it to act as a DFW for that network. It will also contain information about the specific networks that this node is authorized to speak on their behalf as explained by the following equation. 


$$
\begin{aligned}
& \mathrm{IP}_{\text {addresses }} \mathrm{IP} \text { address } \| \text { Subnet mask } 1 \| \text { IP address }{ }_{2} \| \text { Subnet mask } 2 \| \ldots \\
& \text {.... || IP addressn } \| \text { Subnet mask } \\
& \mathrm{C}_{\mathrm{n}}=\mathrm{IP} \text { addresses }\left\|\mathrm{K}_{\mathrm{npb}}\right\| \mathrm{E}\left(\mathrm{H}\left(\mathrm{IP} \text { addresses } \| \mathrm{K}_{\text {npb }}\right), \mathrm{K}_{\text {capp }}\right)
\end{aligned}
$$

where $\mathrm{E}(.$.$) and \mathrm{H}(.$.$) are the encryption and hashing operation respectively.$ Every time the firewall broadcasts a rule, it attaches this certificate to it. On the other hand, when another firewall receives this message it checks the network addresses within the message, against the ones stated in the digital certificate to verify that the sender of the message is authorized to speak on behalf of the networks associated with the rule.

Every time a firewall broadcasts a rule $\mathrm{R}$, it concatenates its digital certificate $C_{n}$ to it. It then, calculates the hash value and encrypts the hash result using its private key $\mathrm{K}_{\mathrm{npr}}$. The output of the encryption process is the digital signature of the rule SIG.

$$
\mathrm{SIG}=\mathrm{E}\left(\mathrm{H}\left(\mathrm{R} \| \mathrm{C}_{\mathrm{n}}\right), \mathrm{K}_{\mathrm{npr}}\right)
$$

Finally, the message $M_{n}$ is constructed by concatenating SIG, R and $C_{n}$ before it is ready in its final form to be broadcasted.

$$
\mathrm{M}_{\mathrm{n}}=\mathrm{R}\left\|\mathrm{C}_{\mathrm{n}}\right\| \mathrm{SIG}
$$

i.e. $\quad M_{n}=R\left\|C_{n}\right\| E\left(H\left(R \| C_{n}\right), K_{n p r}\right)$

When a firewall receives an incoming message $M_{n}$, it extracts the rule $R$, the Digital Signature SIG, the digital certificate $C_{n}$ from the message. Using the certificate $C_{n}$, it extracts the sender's pubic key $K_{n p b}$. The digital signature SIG is decrypted using the public key $\mathrm{K}_{\mathrm{npb}}$, to produce the hash value $\mathrm{H}_{1}$ that was calculated by the sender.

$$
\mathrm{H}_{1}=\mathrm{D}\left(\mathrm{SIG}, \mathrm{K}_{\mathrm{npb}}\right)
$$

Afterwards, it hashes the concatenation of $\mathrm{R}$ and $\mathrm{C}_{\mathrm{n}}$ to calculate $\mathrm{H}_{2}$.

$$
\mathrm{H}_{2}=\mathrm{H}\left(\mathrm{R} \| \mathrm{C}_{\mathrm{n}}\right)
$$

The two hash values $\mathrm{H}_{1}, \mathrm{H}_{2}$ are compared; if they are identical, the sender is authenticated, the message integrity is not compromised, and the rule is accepted for further processing.

To resist the replay attack, the message generated before will be enhanced by adding a time-stamp field $\mathrm{T}_{\mathrm{s}}$ to the rule at the originator. That field will take part of the message generation, as follows:

$$
\begin{gathered}
\quad S I G=E\left(H\left(T_{s}\|R\| C_{n}\right), K_{n p r}\right) \\
M_{n}=T_{s}\|R\| C_{n} \| S I G \\
\text { i.e. } M_{n}=T_{s}\|R\| C_{n} \| E\left(H\left(T_{s}\|R\| C_{n}\right), K_{n p r}\right)
\end{gathered}
$$

On the other hand, the receiver checks this time-stamp against its own clock; if this message was sent before or not. If yes, it will be discarded. By using those three techniques, the protocol is capable of withstanding the threats described earlier. Figure 1 shows the protocol message format and the access control and rule signature are parts of it.

The CDFP has three different rule formats. Rules of level one are concerned with router-based packet filtering firewalls, which contain simple packet filters. Level two rules are more concerned with circuit level proxies, 
and some router-based firewalls that understand transport layer TCP protocol. Level three rules are focused towards today's sophisticated firewalls that contain application layer proxy servers, and can filter application specific traffic. Figure 2 shows CDFP rule formats.

At the defender firewall, DFW, it is the administrator responsibility to insure the proper order of these rules. At the AFW, the order field is defined to resolve any conflicts when they receive rules from the same source. The DFW's are responsible for assigning the right value for this field based on its original table of rules. The AFW's will not be confused by the order of these rules and their priorities because the DFW has already sorted them out. Other mechanism also can be considered such as Hazelhust's algorithm [5].

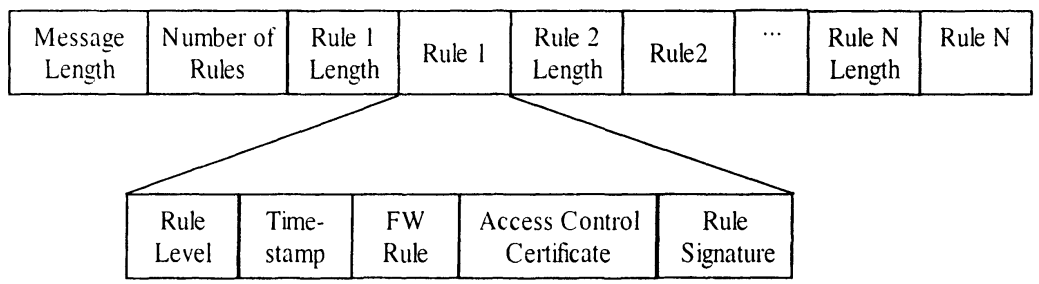

Figure1 CDFP Message Format

\begin{tabular}{|c|c|c|c|c|}
\hline $\begin{array}{c}\text { Rule Level } \\
1\end{array}$ & Order & Src IP & Dest IP & Action \\
\hline
\end{tabular}

(a) Rule format for network layer

\begin{tabular}{|c|c|c|c|c|c|c|c|}
\hline $\begin{array}{c}\text { Rule Level } \\
2\end{array}$ & Order & Src IP & $\begin{array}{c}\text { Dest } \\
\text { IP }\end{array}$ & $\begin{array}{c}\text { Transport } \\
\text { Protocol }\end{array}$ & $\begin{array}{c}\text { Src } \\
\text { Port }\end{array}$ & $\begin{array}{c}\text { Dest } \\
\text { Port }\end{array}$ & Action \\
\hline
\end{tabular}

(b) Rule format for transport layer

\begin{tabular}{|c|c|c|c|c|c|c|c|c|c|}
\hline $\begin{array}{c}\text { Rule Level } \\
3\end{array}$ & Order & $\begin{array}{c}\text { Src } \\
\text { IP }\end{array}$ & $\begin{array}{c}\text { Dest } \\
\text { IP }\end{array}$ & $\begin{array}{c}\text { Transport } \\
\text { Protocol }\end{array}$ & $\begin{array}{c}\text { Src } \\
\text { Port }\end{array}$ & $\begin{array}{c}\text { Dest } \\
\text { Port }\end{array}$ & $\begin{array}{c}\text { Application } \\
\text { Protocol }\end{array}$ & $\begin{array}{c}\text { Application } \\
\text { Sub-Command }\end{array}$ & Action \\
\hline
\end{tabular}

(c) Rule Format for application level

Figure 2 CDFP Rule Formats

\section{SIMULATION AND EXPERIMENTAL RESULTS}

\subsection{Simulator Architecture}

The network simulator we created for this experiment is a $\mathrm{C}++$ application designed using the Object Oriented Programming (OOP) 
concepts [6-8]. It consists of 5 types of objects (classes). The main object is the node object, which simulates a physical firewall, router, or host node. Each node has a number of interfaces that can be linked to other interfaces on other nodes. These links represent the communication links between the nodes in real life. We also implement the Routing Information Protocol RIP [9] to provide the nodes with the capability to dynamically select their own routes between any two nodes without external guidance. We also created a link failure feature to drop any desired link. At that time, the RIP will help selecting an alternative route automatically.

We created a specific high-level configuration language that is used to describe the topology of the network we want to simulate. The simulator also has other types of object that represent different features to enhance the capabilities of each node. Figure 3 shows the simulator architecture.

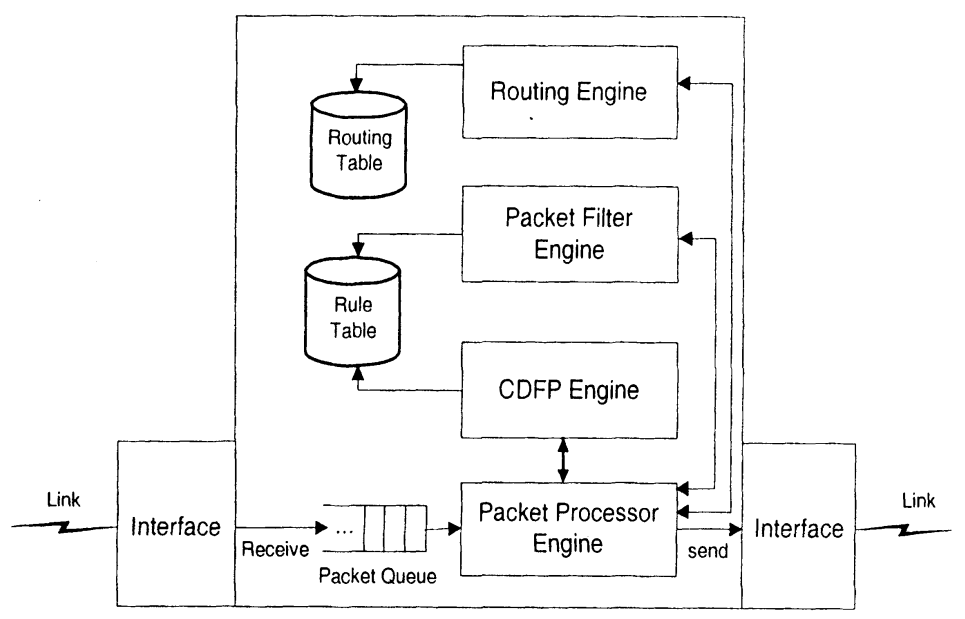

Figure 3 Simulator node object architecture

\subsection{CDFP in Operation}

We conduct our experiment on a matrix of 16 firewalls/router nodes and 2 hosts. As shown in Figure 4-a, Node 17 represents the first host, which simulates the attacker. Node 16 represents the second host, which simulates the target we would like to defend. The DFW is presented in node 15 . The rest of the nodes are AFWs. In all firewall nodes, the Routing Engine and the CDFP Engine are enabled and the routing tables are dynamically built and maintained over time. A packet generator within the node 17 , which generates outgoing traffic to node 16 , simulates the attack. 


\subsection{Example of CDFP Operation}

Consider the above mentioned topology where node 17 represents the attacker, node 16 represents the target, and all other nodes have multiple interfaces so that they have routing and packet filtering capabilities. According to the simulator results, a packet travels from node 17 to node 16 can go through the path $(17,0,1,6,7,11,15,16)$ as shown in Figure $4-a$. When a packet arrives to node 15, it is filtered according to the "deny". This rule is marked as an active rule. The rule is broadcasted from node 15 to the surrounding nodes, nodes 11 and 14 through its interfaces.

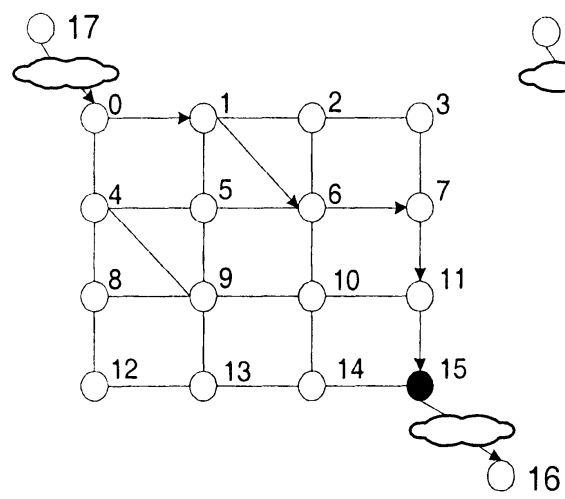

(a)

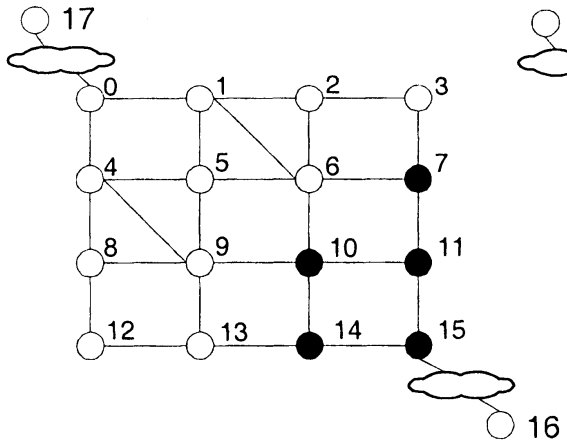

(c)

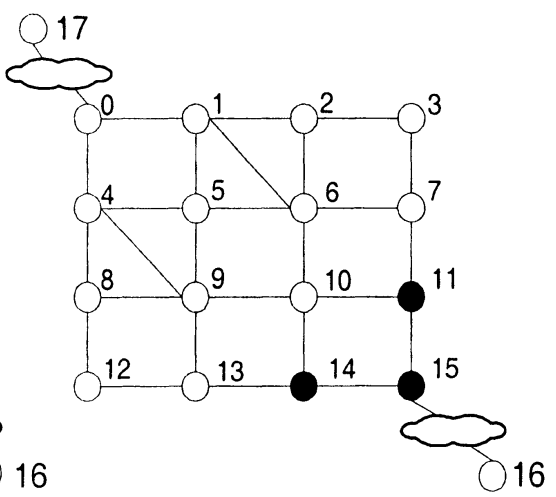

(b)

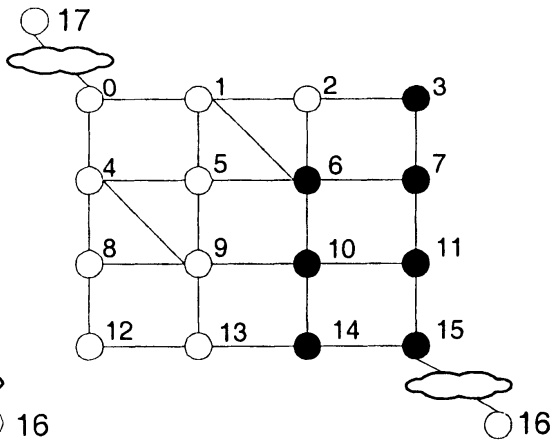

(d)

Figure 4 Experiment : Isolating Attack (Solid nodes show the propagation of FW rules)

The rule continues to propagate towards node 17 as long as the traffic continues as shown in Figure 5. Node "0" keeps filtering the attack traffic as long as node 17 is attacking. It also broadcasts the rule to node 4 as shown in Figure 5-c. Other nodes hold the rule for certain time, after that they discarded the rule as shown in Figure 5-d. By filtering the traffic earlier at 
node " 0 ", the attacker is isolated far from the target node 16 . The CDFP is able to apply the security policy at further points within the path of attack and to isolate the attack at the nearest point to the attacker.

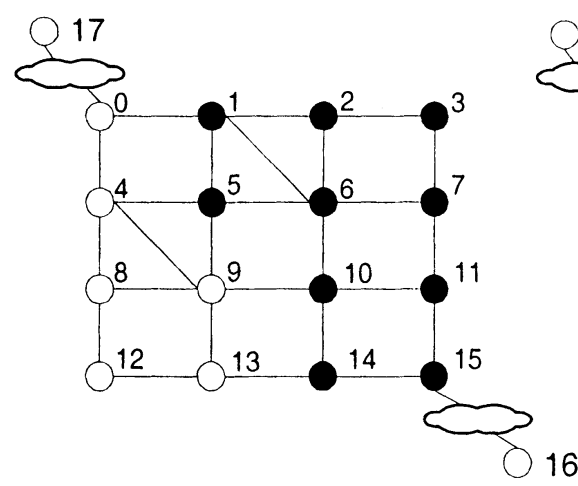

(a)

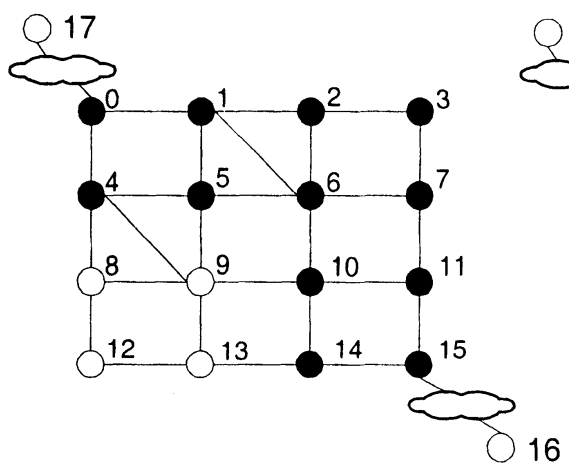

(c)

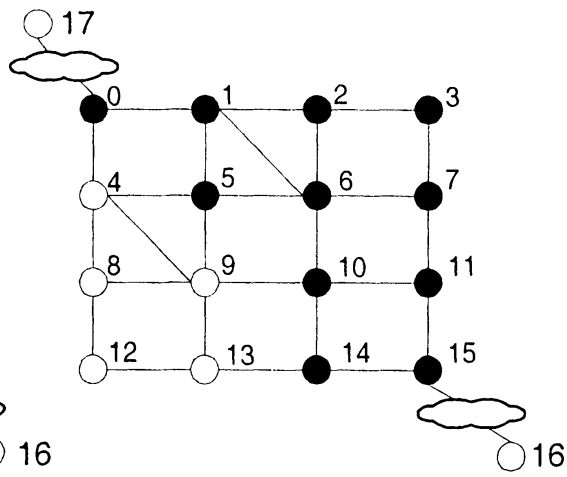

(b)

Figure 5 Isolating Attack (continued)

(Solid nodes show the propagation of FW rules)

\subsection{Behavior during Route Change and Network Topology Change}

The attacker can change the path of the attack by using the source route option within the IP protocol. If the route changes before the rule reaches the firewall at the attacker's network and isolates the traffic of the attack, the attack will take its route to the defender firewall. The defender firewall will detect the traffic from the new path and will start broadcast its messages towards the new path. The cooperation relations will be built with the 
firewalls within the new path. The same is true in case of a change in network topology.

\subsection{Behavior during IP Spoofing Attack}

During IP Spoofing attacks the attacker impersonates himself as a different node on the network. Within this attack, there are two different paths; one is the physical path of attack, which starts by the real source towards the target destination. The second one is the virtual path of attack, which starts by the impersonated node, towards the target destination. As before, the CDFP protocol will detect the real path of attack, and broadcast the "deny" rule towards that path. This will result in isolating the attacker by the nearest available firewall. Other experiments have been conducted to study the behavior of the proposed protocol. The details are given in [10].

\section{CDFP VERSUS CGFW}

Both CGFW and CDFP main goal is to isolate the attacker to the farthest possible point, each does this in a different manner. As mentioned before, the CGFW is built on top of BGP-4 routing protocol which has a database with details about the interconnected networks topology. This database is dynamically updated based on the network status. When a node is subject to an attack, the CGFW uses this information to calculate the optimum nodes that are used to partition the network into a secure zone and insecure zone. Then, it sends the filter request to these specific nodes to ask them to cooperate in the defense process. Although CGFW uses the same graph theory used in ATM networks [11], partitioning the network to isolate the attacker is a difficult task in case of a wide network such as the Internet with a very complex topology [1]. On the other hand, CDFP uses broadcasting to deliver the message to the surrounding nodes, which will hopefully create an immediate shell to protect it. In turn, those nodes will deliver the message to their neighbors in the path of attack and thus, CDFP reaches the optimum points.

Concerning the security features such as authentication, BGP-4 has an optional authentication feature, which can be turned on by administrators. The authentication feature is presented in using the Message Authentication Code (MAC). The technique uses a one-way hash function (usually MD5) with a key to generate a 16-bytes value at the sender to be transmitted with the message. The receiving end must have the same key to be able to verify the sender identity and the message integrity.

Both CGFW and BGP-4 specifications have not defined an access control mechanism [1]. So, if someone passes the authentication check, he is free to distribute information about other portions of the network. On the 
other hand, CDFP can use digital certificate and access control mechanism based on the RSA public key algorithm.

CGFW has a time limits in the filter request. The filter and monitor requests will begin based on a start time and last for a specified duration. CDFP has also a time-stamp field as a replay attack detection mechanism.

Concerning the operation requirements, CGFW processing power is mainly consumed in the search process to find the optimum points. That can be a lengthy search if the number of nodes is high. The calculation process will also depend on the attacker's source address, which could be easily spoofed in some attacks. The routing table could be huge if the network is too complex and a long processing time could be expected while calculating the optimum nodes to be contacted. Also, CGFW needs to maintain a table for the network topology and a second table for the firewall rules. It worth to mention that the CGFW processing could be used as a DoS by keeping the router node busy trying to calculate the optimum points. In the case of CDFP, most of the processing power is consumed in the cryptographic operations to sign or verify the messages. CDFP needs only one table to hold the firewall rules, the process of broadcasting is very light in comparison to the CGFW, but it requires more memory for the certificates and other security information.

CGFW has a network topology database that has to be updated through the exchange of messages between different nodes. These messages will be exchanged only when a change in the network occurs, and will be communicating to the calculated nodes directly during an attack. While CDFP does not need a lot of traffic to initialize, it will consume more bandwidth during an attack because of the broadcasting. It needs to retransmit the message to the nodes within the path of attack and their neighbors till it reaches the optimum point. Usually the number of retransmission for a filter request is proportional to the number of intermediate nodes between the attacker and the target.

\section{DISCUSSION AND CONCLUSIONS}

The proposed CDFP protocol is designed specifically to achieve cooperative communication between firewalls during attacks. This protocol enables firewalls to advise each other about current and potential malicious activities on the network, which provides firewalls with more intelligence to help withstand the increasing number of sophisticated attacks on today's networks. By acquiring this cooperative defense approach between firewalls, we can further isolate the attacker from reaching the target network by filtering its access from the nearest available firewall node to this network. Thus, this can help preventing unnecessary traffic and save bandwidth. On the other hand, hackers could use CDFP to perform a denial 
of service attack. To overcome this risk, security measures such as authentication, access control, message integrity, and replay detection, are added to CDFP. We use a network simulator, built using $\mathrm{C}++$, to study CDFP behavior during isolating a direct attack, IP Spoofing attack, network topology change and security policy change. In case of an attack within the CDFP own network, the protocol succeeded in filtering the traffic at the nearest node to the attacker, isolating it far from the target node. We also found that CDFP protocol is able to deliver the policy change to all the other nodes which are able to apply the "permit" rule. The same is true in case of network topology change since it can track the dynamically changing path of attack. Throughout the experiments conducted using our simulator, we strived to simulate as much as possible the real life environment where CDFP will exist. In simulation a single CA was used for simple illustration. In more complex network scenario, digital certificate chaining concepts of PKI could be employed. The PKI technology is designed to be scaleable. It allows Certificate Authorities to be chained. Finally, there were two objectives that we excluded from our experiments for the sake of simplicity. One is implementing the cryptographic portion within the CDFP protocol, and the second is running the protocol between firewalls with different levels of intelligence or within different autonomous systems. This will be the subject of future work.

\section{REFERENCES}

[1] Robert N. Smith and Sourav Bhattacharya, "A Protocol and Simulation for Distributed Communicating Firewalls", in Proceedings of IEEE $23^{\text {rd }}$ Annual International Computer Software and Applications Conference, COMPSAC, pp:74-79,1999.

[2] Chris Hare and Karanjit Siyan, "Internet Firewalls and Network Security (Second Edition)", New Riders Publishing, Indiana Polise, IN, 1996.

[3] "Maximum Security: A Hacker's Guide to Protecting Your Internet Site and Network (Second Edition)", Book News, Inc., Portland, 1999.

[4] Rekhter, Y. and Wastson, T.J., Li., "A Border Gateway Protocol 4 (BPG-4), Internet Engineering Task Force”, Network Working Group, RFC-1771, March 1995.

[5] Scott Hazelhust, Raymond Sinnappan and Adi Attar, "Algorithms for Improving the Dependability of Firewall and Filter Rule Lists", Proceedings of the International Conference on Dependable Systems and Networks (DSN 2000), IEEE COMPSAC, pp:576-585, 2000.

[6] Jim Holmes, "Building Your Own Compiler With C++", Prentice Hall, Englewood Cliffs, NJ, 1995.

[7] John Lakos, "Large-Scale C++ Software Design", Addison-Wesley Publishing Company, 1996.

[8] Scott Meyers, "Effective C++ (Second Edition)", Addison-Wesley Publishing Company, 1997.

[9] Chuck Hedrick, "Routing Information Protocol", RFC 1058, June 1988.

[10] Mohamed A. Eissa, "Coperative Defence Firewall Protocol", M. Sc. Thesis, Faculty of Engineering, Cairo University, January 2002.

[11] Rainer, R., MacGregor, M.H., Grover, W.D., "Optimal Capacity Placement for Path Restoration in STM or ATM Mesh-Survivable Networks", IEEE/ACM Transactions on Networks, Vol.6, No.3, pp:325-336, June 1998. 